\title{
Imágenes en endocarditis infecciosa: No todo es ecocardiografía
}

\author{
Imaging in infective endocarditis: Not all is ultrasonography
}

David Ladrón-de-Guevara H. ${ }^{1,2}$, Alejandro Canelo L. ${ }^{3}$, Patricia Bitar H. ${ }^{1}$ y Juan Ramón Soto S..$^{2,4}$

'Departamento de Radiología, Clínica Las Condes. Santiago, Chile.

${ }^{2}$ Facultad de Medicina Universidad de Chile. Santiago, Chile.

${ }^{3}$ Interno de Medicina, Facultad de Medicina Pontificia Universidad Católica de Chile. Santiago, Chile.

${ }^{4}$ Departamento de Cardiología, Clínica Las Condes. Santiago, Chile.

Los autores declaran no tener conflictos de interés.

No se recibió financiamiento alguno asociado a este manuscrito.

Recibido: 1 de agosto de 2020 / Aceptado: 5 de febrero de 2021

\section{Resumen}

La endocarditis infecciosa (EI) es una enfermedad de alta mortalidad, caracterizada por una infección endocárdica y frecuentes complicaciones multiorgánicas, que requiere un diagnóstico rápido y preciso, y un manejo agresivo, ya sea médico o quirúrgico. $\mathrm{Su}$ diagnóstico se realiza tomando en cuenta criterios bacteriológicos, clínicos y ecocardiográficos. Es objetivo de este artículo realizar una actualización del estudio imagenológico en paciente con EI, con especial énfasis en aquellos exámenes no ecocardiográficos disponibles en nuestro medio. En los últimos años, estudios de imagen avanzados han adquirido un rol creciente en su estudio inicial, particularmente la tomografía computada multicorte (TCMC) cardiaca y el positron emission tomography/computed tomography (PET/CT), y han sido recomendados como criterios diagnósticos en las guías recientes para el manejo de esta entidad. La TCMC cardiaca proporciona información anatómica detallada de las válvulas cardiacas y tejido perivalvular, identificando pseudoaneurismas, abscesos y dehiscencias valvulares. E1 PET/CT con F18-fluorodeoxiglucosa (F18-FDG) permite aumentar la sensibilidad en la detección de EI, y pesquisar con alta eficiencia fenómenos embólicos sistémicos, de elevada frecuencia en esta población. Ambos métodos prestan particular utilidad en EI de válvula protésica, donde la ecocardiografía presenta menor rendimiento diagnóstico. La resonancia magnética (RM) cerebral es el mejor método de imagen para descartar eventos isquémicos/embólicos del sistema nervioso central.

Palabras clave: endocarditis infecciosa; PET/CT; tomografía computada cardiaca; prótesis valvular; dispositivos cardiacos.

\section{Abstract}

Infective endocarditis (IE) is an entity characterized by endocardial infection and frequent multiorgan complications, resulting in high mortality. It requires a rapid and accurate diagnosis, and a medical or surgical aggressive treatment. Currently, IE diagnosis rests on bacterial, clinical and ultrasonographic criteria. The objective of this article is to update the imaging study in patients with IE, with special emphasis on those non-echocardiographic examinations available in our environment. Last years, advanced imaging had achieved a growing role in IE diagnosis, especially cardiac multislice computed tomography (MSCT) and positron emission tomography/computed tomography (PET/CT), which have been recommended in recent clinical guidelines to be included as part of diagnostic criteria. Cardiac MSCT provides detailed anatomic information of cardiac valves and perivalve tissue, allowing identification of pseudoaneurysm, abscess and valve dehiscence. F18-FDG PET/CT increases sensitivity for IE detection and shows high accuracy in searching for extracranial systemic embolic events. Both MSCT and PET/CT have particular utility in cases of prosthetic valve endocarditis, where cardiac ultrasonography shows lower performance. Brain magnetic resonance imaging (MRI) is the best imaging method for evaluating ischemic/embolic events of central nervous system.

Keywords: infective endocarditis; 18F-FDG-PET/CT; cardiac computed tomography; prosthetic valves; cardiac devices.

\section{Correspondencia a:}

David Ladrón de Guevara H.

dlg@clc.cl 


\section{Criterios diagnósticos actuales}

a endocarditis infecciosa (EI) se define como una infección de las válvulas cardíacas nativas o protésicas, la superficie endocárdica no valvular del corazón, de las arterias o de dispositivos cardíacos artificiales, tales como los electrodos de un marcapasos, entre otros. Pese al advenimiento de nuevas estrategias diagnósticas y terapéuticas, la mortalidad a un año no ha cambiado y permanece en torno a $30 \%$, es decir, mayor que la de muchos cánceres ${ }^{1}$.

La EI es una afección clínica infrecuente, teniendo una incidencia anual de 3-10 casos por 100.000 personas ${ }^{2}$. Las secuelas valvulares de la cardiopatía reumática, las valvulopatías degenerativas (fibrocálcica), mixomatosis valvular y cardiopatías congénitas son sustratos que pueden predisponer a la infección bacteriana ${ }^{3,4}$. Actualmente, se estima que hasta $30 \%$ de las EI son adquiridas en el ámbito de atenciones de salud -tanto intrahospitalarias como ambulatorias- contribuyendo a esto el uso de líneas endovenosas durante un tiempo prolongado, hemodiálisis, procedimientos invasivos y, el uso de dispositivos artificiales cardíacos, como marcapasos, re-sincronizadores y desfibriladores ${ }^{5}$.

En Chile Oyonarte y cols. ${ }^{6}$, han reportado el seguimiento prospectivo de una cohorte de más de 500 pacientes con el diagnóstico de EI (período comprendido entre los años 1998-2008). Este estudio mostró que en nuestro país la enfermedad valvular reumática es el sustrato predisponente más frecuente $(22 \%)$, siendo seguido de cerca por las prótesis valvulares (14\%) y las cardiopatías congénitas (14\%).

La EI en válvula protésica es la forma más grave de EI y constituye hasta $30 \%$ del total de casos $^{7,8}$. Se presenta en hasta $4 \%$ de los pacientes dentro de los cinco primeros años de la cirugía índice y compromete en igual proporción a las prótesis mecánicas y biológicas ${ }^{9}$. El diagnóstico es difícil puesto que las manifestaciones clínicas suelen ser atípicas y las imágenes ecocardiográficas pueden resultar falsamente negativas en una frecuencia relativamente alta, lo que determina que los criterios de Duke resulten insuficientes para sustentar la hipótesis diagnóstica ${ }^{10}$.

En los últimos años, se ha modificado significativamente la manera de estudiar a estos pacientes, con un creciente rol de las imágenes en el proceso diagnóstico. Consensos actuales incorporan a la tomografía computada multicorte (TCMC) cardiaca y al positron emission tomography/computed tomography (PET/CT) utilizando F18-fluorodeoxiglucosa $\left({ }^{18} \mathrm{~F}-\mathrm{FDG}\right)$ entre los criterios diagnósticos?

El objetivo de este trabajo es realizar una actualización del estudio imagenológico en paciente con EI, con especial énfasis en aquellos exámenes no ecocardiográficos disponibles en nuestro medio.
Para el diagnóstico de EI se han utilizado ampliamente los criterios de Duke, cuya última modificación data del año $2000^{11}$. Dichos criterios integran hallazgos clínicos, microbiológicos e imagenológicos, estos últimos basados exclusivamente en ecocardiografía trans-torácica (ETT) y trans-esofágica (ETE). Estos criterios clasifican el diagnóstico de EI como definitivo, posible o rechazado, basados en el cumplimiento de criterios mayores y menores (Tabla 1).

Las guías para el manejo de la EI de la Sociedad Europea de Cardiología (ESC 2015), publicadas en 2015, han propuesto la incorporación de la TCMC cardíaca, PET/ CT con ${ }^{18} \mathrm{~F}-\mathrm{FDG}$ y SPECT/CT con leucocitos marcados, como criterios diagnósticos mayores independientes, y la demostración mediante imágenes de eventos embólicos silentes como un criterio menor ${ }^{7}$. Estos exámenes de imagen de última generación ya están siendo incorporados en los criterios diagnósticos en la literatura médica actual ${ }^{12}$. Los nuevos criterios propuestos se muestran como anexos de los criterios modificados de Duke en la Tabla 1.

El tratamiento de la EI comprende terapia antimicrobiana y, en determinadas circunstancias, la cirugía de reparación o reemplazo valvular.

\section{Complicaciones}

Las complicaciones más graves de la EI (insuficiencia cardíaca, infección no controlada y embolías) suelen ser de tratamiento quirúrgico. La complicación más común es la falla cardiaca congestiva como resultado de la destrucción valvular y su consiguiente insuficiencia ${ }^{7,13}$. Ocurre en 42 a $60 \%$ de las EI de VN, y se asocia más frecuentemente a IE aórtica? ${ }^{7}$.

Por otra parte, complicaciones inflamatorias e inmunológicas, tales como la glomerulonefritis por complejos inmunes, mialgias y tenosinovitis, son frecuentes en pacientes con $\mathrm{EI}^{14}$.

La embolización sistémica afecta a aproximadamente un tercio de los pacientes con EI y tiene un impacto negativo en su mortalidad ${ }^{15}$. Las embolias del sistema nervioso central suelen ser las más ominosas, aunque pueden también generarse infartos a nivel renal, esplénico, coronario, y otros sistemas. La extensión de la infección a otros tejidos mediante embolias sépticas, como ocurre en los pseudo-aneurismas micóticos (embolias sépticas de la vasa vasorum), también merece ser considerada ${ }^{7,13}$.

La embolia osteo-articular, y particularmente la espondilodiscitis, es una de las complicaciones más frecuentes, con una incidencia de 2,6 a 19\% en pacientes con diagnóstico de $\mathrm{EI}^{16,17}$. Durante los últimos años, la pesquisa de espondilodiscitis y otros focos embólicos no 
Tabla 1. Criterios modificados de Duke para el diagnóstico de endocarditis infecciosa ${ }^{11}$, complementados de acuerdo a los nuevos criterios propuestos por la Sociedad Europea de Cardiología 20157

\section{Diagnóstico definitivo}

Criterios anatomo-patológicos: microorganismos identificados por cultivo o examen histológico de una vegetación, de una vegetación que ha embolizado, o de un absceso intracardiaco; o endocarditis activa confirmada por examen histológico de una vegetación o absceso intracardiaco

Criterios clínicos: 2 mayores, 1 mayor y 3 menores, o 5 menores

\section{Criterios clínicos mayores}

1. Hemocultivo positivo para El

a. Microorganismos típicos consistentes con El en 2 hemocultivos separados: Streptococcus grupo viridans, Streptococcus bovis, bacterias del grupo HACEK**, o Staphylococcus aureus; o Enterococcus spp adquiridos en la comunidad en la ausencia de un foco primario

b. Microorganismos consistentes con El identificados en hemocultivos persistentemente positivos: hemocultivos positivos de al menos dos muestras extraídas con $>12 \mathrm{~h}$ de diferencia; o de tres o la mayoría de cuatro o más hemocultivos (con la primera y última muestra extraídas al menos con 1 hora de diferencia); o un único hemocultivo positivo para Coxiella burnetii o títulos de anticuerpos lgG fase I > 1:800

\section{Evidencia de compromiso endocárdico}

a. Ecocardiografía positiva para El: vegetación (masa oscilante) valvular o en estructuras de apoyo, absceso, o nueva dehiscencia parcial de válvula protésica;

b. Nueva insuficiencia valvular

3. Imagen positiva para El en $\mathrm{VP} * 7$

a. PET/CT ${ }^{18} \mathrm{~F}$-FDG con captación anormal en la zona de implantación de la válvula protésica (sólo si la prótesis fue implantada hace más de 3 meses) ó SPECT/CT con aumento de captación de leucocitos marcados

b. Lesión paravalvular categórica en TCMC cardiaca

\section{Criterios clínicos menores}

a. Predisposición a El, como enfermedad cardiaca predisponente o uso de drogas intravenosas

b. Fiebre $>38^{\circ} \mathrm{C}$

c. Fenómenos vasculares, como embolia arterial mayor, infartos sépticos pulmonares, pseudo-aneurisma micótico, hemorragia intracraneal, hemorragia conjuntival, y lesiones de Janeway

d. Fenómenos inmunológicos, como glomerulonefritis, nódulos de Osler, manchas de Roth, y factor reumatoide

e. Evidencia microbiológica: hemocultivo positivo, pero sin cumplir un criterio mayor o evidencia serológica de infección activa por un organismo consistente con El

f. Fenómenos embólicos recientes o pseudo-aneurismas infecciosos identificados sólo por imágenes (eventos silentes)*

\section{Diagnóstico posible}

Criterios clínicos (ver arriba): 1 criterio mayor y 1 criterio menor, o 3 criterios menores

\section{Diagnóstico descartado}

Diagnóstico alternativo firmemente establecido; resolución del síndrome de "endocarditis infecciosa-like" con terapia antimicrobiana durante $\leq 4$ días; ausencia de evidencia anatomopatológica de El en cirugía o autopsia, con antibioterapia durante $\leq 4$ días; o criterios de El posible no cumplidos

*En azul los criterios agregados según el consenso de la Sociedad Europea de Cardiología $2015^{7}$. El: endocarditis infecciosa; HACEK: Haemophilus spp., Aggregatibacter spp, Cardiobacterium hominis, Eikenella corrodens, Kingella spp; VP: válvula protésica. sospechados clínicamente ha aumentado gracias al uso de técnicas de imagen de cuerpo completo, particularmente el PET/CT ${ }^{18}$.

\section{Imágenes en endocarditis infecciosa}

\section{Ecocardiografía}

La ecocardiografía es la técnica de imágenes de primera elección para el diagnóstico de EI. Tres hallazgos ecográficos forman parte de los criterios mayores de diagnóstico de EI: compromiso del endocardio con vegetaciones; extensión perivalvular de la infección; y signos de afectación de la integridad valvular en una válvula nativa o protésica ${ }^{11}$.

El primer método de imágenes que se suele usar ante la sospecha de EI es la ETT. Avances tecnológicos producidos durante los últimos veinte años han permitido mejorar su resolución espacial, de tal forma que su sensibilidad para detectar vegetaciones en válvulas nativas está entre 80 y $89 \%{ }^{19}$. Su especificidad ha sido reportada en el rango de 70 a $90 \%{ }^{20}$.

La ETE no tiene las limitaciones que una ventana acústica deficiente impone en ocasiones a la ETT. Dada la proximidad del transductor al corazón, las imágenes pueden adquirirse con una alta frecuencia de emisión del ultrasonido lo que permite obtener imágenes de excelente resolución espacial, pudiendo detectar vegetaciones tan pequeñas como de $2 \mathrm{~mm}$. El advenimiento de la ETE tridimensional ha contribuido a su elevada precisión diagnóstica. En válvulas nativas la sensibilidad de la ETE es cercana a $100 \%$ y su especificidad supera el $90 \%{ }^{20,21}$.

La endocarditis en válvula protésica es difícil de detectar mediante ETT, en parte debido a que las vegetaciones son menos frecuentes (60-70\%) y, en cambio, es más frecuente el compromiso perivalvular, de difícil visualización mediante ETT. Esto determina que la sensibilidad de la ETT en este contexto sea menor a $50 \%{ }^{7}$. En cambio, frente a la sospecha de EI en válvula protésica, la ETE tiene un rendimiento diagnóstico claramente superior, con una sensibilidad en el rango de 80 a $95 \%$ y especificidad superior a $90 \%$, lo que lo convierte en la técnica ecográfica de primera elección en este escenario ${ }^{22}$.

A pesar del buen rendimiento de la ETE, su capacidad diagnóstica no es absoluta, particularmente en el contexto de la EI en válvula protésica, y dada la alta mortalidad de la EI se ha hecho necesario el desarrollo de otros métodos diagnósticos, dentro de los cuales la TCMC y las imágenes híbridas se han posicionado como una alternativa prometedora.

\section{Tomografía computada multicorte cardíaca}

La TCMC ha sido incluida recientemente en los criterios diagnósticos de EI de la Sociedad Europea 
de Cardiología (ESC 2015)7 , y consiste en una TCMC gatillada. La demostración de una lesión paravalvular mediante TCMC cardiaca constituye un criterio diagnóstico mayor de $\mathrm{EI}^{7}$.

La capacidad de evaluar detalladamente la anatomía cardiaca con TCMC está dada por su alta resolución espacial, y además, la alta resolución temporal que se logra al realizarla con gatillado electrocardiográfico, en que se sincroniza la adquisición y reconstrucción de las imágenes con el ECG del paciente obtenido en forma simultánea $^{23}$. De este modo se obtienen imágenes con gran detalle anatómico, con grosor de corte en todos los ejes de aproximadamente $0,4 \mathrm{~mm}$; y con alta velocidad, lo que permite visualizar al corazón quieto, sin artefacto de movimiento cardiaco.

Existe la posibilidad de realizar una TCMC con adquisición prospectiva, en que sólo se obtienen las imágenes en la fase del ciclo cardiaco en que el corazón está más quieto para evaluar anatomía; o con adquisición retrospectiva, en que se obtienen imágenes durante todo el ciclo cardiaco, lo que permite reconstruir al corazón estático, pero además en todo el ciclo y así evaluar función ${ }^{24}$. Para el estudio de EI se prefiere un protocolo retrospectivo que permitirá evaluar la anatomía valvular durante todo el ciclo cardiaco.

El protocolo ideal consiste en dos adquisiciones. Se realiza primero una adquisición prospectiva sin uso de contraste endovenoso que permite evaluar calcificaciones y elementos densos, y programar el largo de la adquisición contrastada. Posteriormente se administra contraste endovenoso con doble inyección (contraste y luego mezcla de contraste con solución salina $[\mathrm{NaCl} 9 \%$ ] estéril a $5 \mathrm{ml} / \mathrm{min}$ ) y se realiza una adquisición retrospectiva sin modulación de dosis. Se obtienen tres reconstrucciones distintas: sin contraste, con contraste en la fase de menor movimiento cardiaco, y con contraste del ciclo cardiaco completo. Con la reconstrucción sin contraste se evalúa las calcificaciones cardiacas, valvulares, pericárdicas, coronarias y también los elementos metálicos o densos de cirugías previas, lo que ayuda a diferenciar patología de cambios postquirúrgicos esperables. Con la reconstrucción del corazón quieto se evalúa toda la anatomía cardiaca, valvular, coronaria, pericárdica. Y con las reconstrucciones dinámicas del ciclo cardiaco completo se evalúa la función cardiaca y valvular, que incluye motilidad, grosor parietal, tamaño de cavidades, entre otros ${ }^{23,24}$.

El uso de TCMC es considerado apropiado para caracterizar válvulas nativas o protésicas cuando se sospecha disfunción significativa, y existiendo imágenes inadecuadas obtenidas por otros métodos no invasivos ${ }^{22}$.

Particularmente en el contexto de EI, con TCMC cardiaca es posible evaluar la anatomía valvular, su movimiento en el ciclo cardiaco, presencia de vegetaciones (Figura 1), y complicaciones de endocarditis como perforación valvular, pseudo-aneurismas/abscesos (Figura 2), dehiscencia valvular, fuga paravalvular.

Un reciente estudio demostró que la detección de pseudo-aneurismas y abscesos por TCMC es mayor que con ecocardiografía, y que la combinación de ambos métodos incrementaría la sensibilidad en hasta $100 \%$. Además, complementar la ETE con TCMC aumentaría la detección de infiltrado inflamatorio ${ }^{25}$. La TCMC no es superior a ecocardiografía en el diagnóstico de vegetaciones, perforación de valvas ni fugas perivalvulares, pero sería útil cuando la ecocardiografía da resultado indetermina$\mathrm{do}^{25}$. Otro estudio demostró que la TCMC cardiaca tiene capacidad diagnostica similar a la ETE para la detección

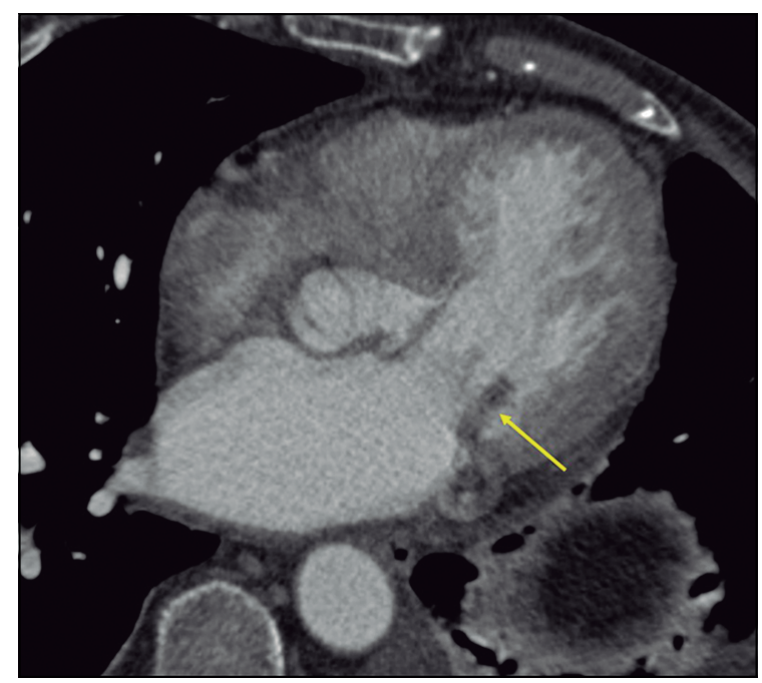

Figura 1. Corte axial de TCMC de tórax no gatillado, que demuestra tejido de densidad de tejidos blandos en la válvula mitral (flecha), que corresponde a vegetación.

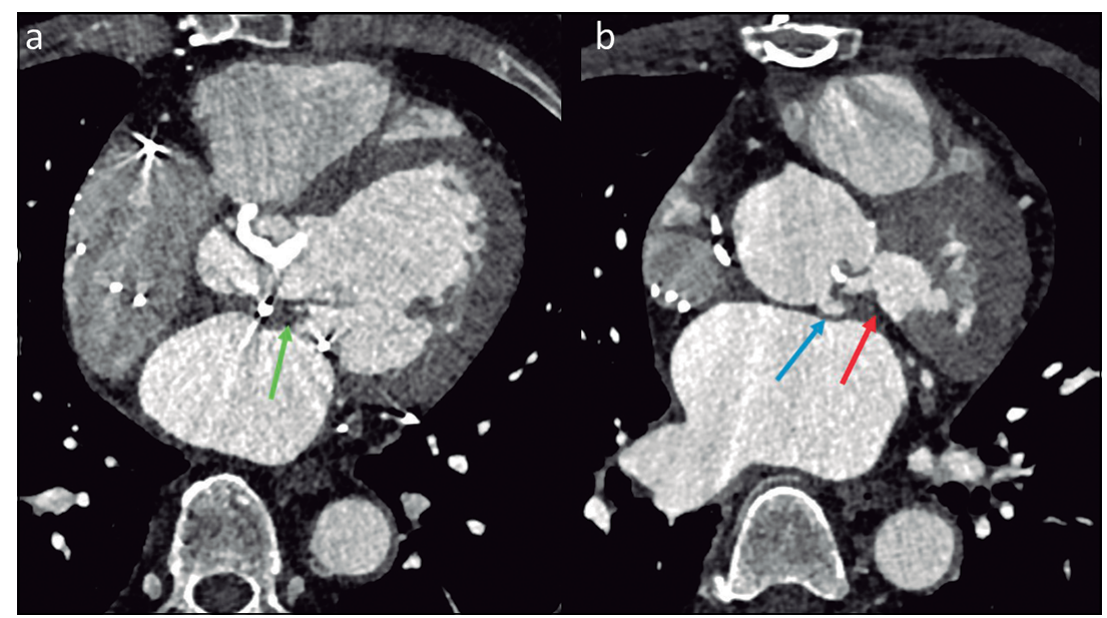

Figura 2. Hombre de 60 años con anillo de valvuloplastía mitral, que posteriormente desarrolla una endocarditis infecciosa. Cortes axiales de TCMC cardiaco gatillado, que demuestran: a) vegetación en anillo mitral (flecha verde); y b) tejido denso inflamatorio que se extiende a la fibrosa intervalvular mitroaórtica (flecha roja) y formación de pequeño pseudo-aneurisma en raíz aórtica (flecha celeste). 


\section{— -}

de vegetaciones grandes y de varias complicaciones de la endocarditis; y que sería superior en la detección de abscesos perivalvulares y enfermedad coronaria ${ }^{26}$.

En caso de EI de cavidades derechas, la TCMC y PET/CT son especialmente útiles, ya que pueden revelar patología pulmonar asociada, como infartos, émbolos sépticos o abscesos pulmonares ${ }^{7}$ (Figura 3).

\section{SPECT/CT}

El SPECT/CT utilizando leucocitos marcados muestra menor sensibilidad (64 vs $93 \%$ ) y mayor especificidad (100 vs 71\%) que el PET/CT en la detección de EI de válvula protésica ${ }^{27}$, aunque existe significativa menor experiencia comparativa publicada sobre $\mathrm{SPECT}^{28}$. Esta menor evidencia puede ser debido a la complejidad técnica del examen, que requiere extracción de 30-40 $\mathrm{ml}$ de sangre, marcación in vitro, y re-inoculación endovenosa de la muestra, lo que es técnicamente demandante y consumidor de tiempo, dificultando su uso en la práctica clínica $^{28-30}$. Nuevos radio-trazadores basados en anticuerpos monoclonales anti-granolucitos prometen simplificar este proceso de marcación ${ }^{29}$, haciendo menos engorroso el examen. Sin embargo, falta evidencia de la utilidad específica de dichos radio-trazadores en el estudio de EI.

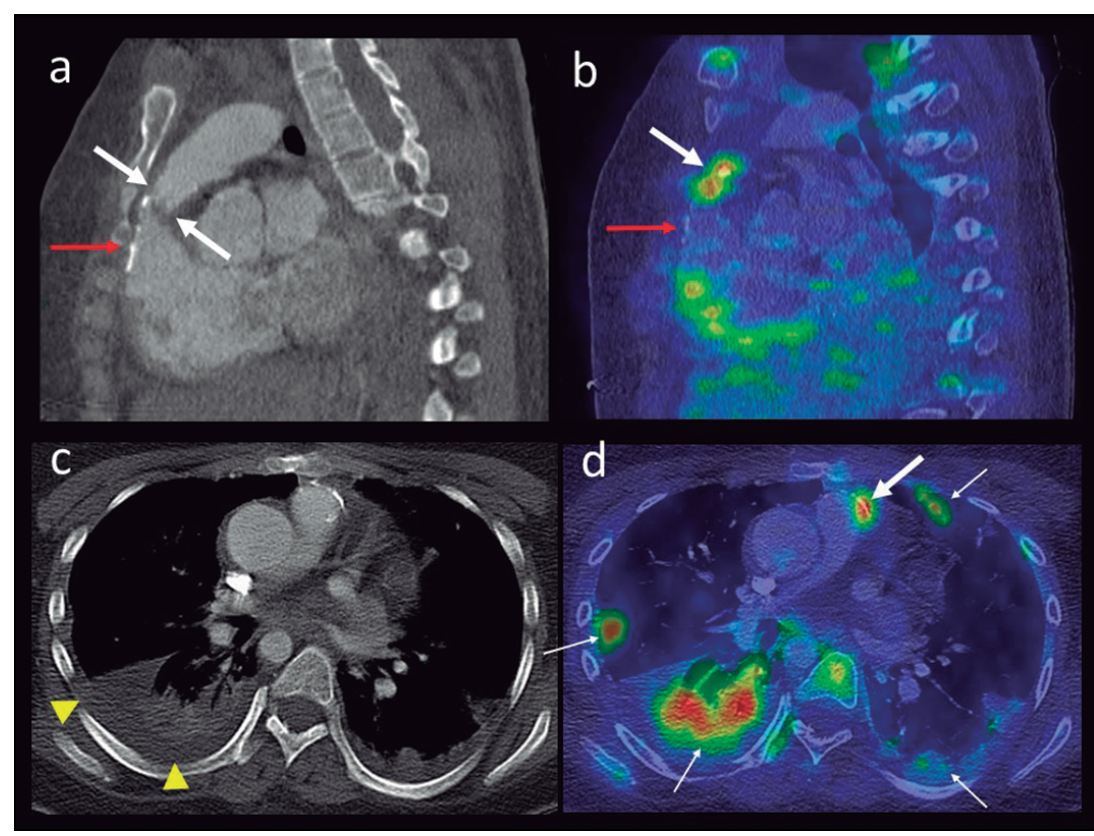

Figura 3. Paciente de 17 años con tetralogía de Fallot (TOF) reparada en la infancia, que desarrolla una endocarditis infecciosa de la válvula pulmonar. Reconstrucción sagital de TCMC cardiaca (a) y PET/CT (b) que muestran engrosamiento de la válvula pulmonar, hipecaptante con SUVmax: 4,0 (flechas blancas), y cambios post-quirúrgicos de reparación de TOF, con calcificación del parche en la superficie anterior del ventrículo derecho, sin captación de F18-FDG (flecha roja). Corte axial de la TC (c) y PET/CT (d) donde se demuestran múltiples embolias sépticas pulmonares bilaterales (flechas delgadas), asociado a efusión pleural derecha (cabezas de flecha amarillas). Foco hipercaptante de endocarditis de válvula pulmonar en flecha blanca gruesa en (d). Hemocultivos positivos para Staphylococcus aureus sensible a meticilina.
Por otro lado, la toma de imágenes de 3-4 h y 20-24 h después de inoculado el radio-trazador ${ }^{29}$ constituye una limitación en aquellos casos en que se requiere tomar decisiones terapéuticas rápidas ${ }^{27}$.

La alta especificidad del SPECT/CT sería particularmente útil en casos con ecocardiografía y PET/CT no concluyentes en pacientes con cirugía cardiaca reciente, es decir dentro del último mes ${ }^{31}$. Una de las razones de la baja sensibilidad del SPECT es su pobre resolución espacial, lo que juega en contra de esta técnica, especialmente si se quiere detectar focos pequeños de captación, esperables en la $\mathrm{EI}^{30}$. En un meta-análisis reciente, Albano y cols., encontraron sólo cuatro series retrospectivas que estudiaban el uso del SPECT en $\mathrm{IE}^{28}$. Los autores coinciden en que se requiere mayor evidencia para conocer el verdadero rol del SPECT en el estudio de IE $^{28,32}$.

\section{PET/CT}

El PET/CT utilizando ${ }^{18} \mathrm{~F}-\mathrm{FDG}$ es de gran utilidad en el estudio de EI y también ha sido incluido recientemente en los criterios diagnósticos de EI de la Sociedad Europea de Cardiología (ESC 2015) ${ }^{7}$. Su aporte principal es disminuir significativamente el número de diagnósticos "posibles", re-clasificándolos como "definitivo" o "rechazado"33. $\mathrm{Su}$ rendimiento diagnóstico depende de una adecuada preparación del paciente, y de si la EI se produce en una válvula nativa o protésica. De hecho, su sensibilidad es significativamente mayor en EI asociadas a válvula protésica.

La intensidad de captación valvular de ${ }^{18} \mathrm{~F}-\mathrm{FDG}$ se asocia al grado de infiltración del tejido por células inflamatorias, tanto respecto al número de células como a su naturaleza. Las células sanguíneas que suelen reclutar estas áreas inflamatorias son los polimorfonucleares, macrófagos y linfocitos, que suelen presentar alta captación de glucosa ${ }^{34}$. De Camargo y cols., demostraron que la intensidad de captación fue más elevada en lesiones con mayor infiltrado inflamatorio y mayor número de polimorfonucleares a la histología ${ }^{34}$.

La intensidad de captación es posible de cuantificar utilizando índices como el SUV (standarized uptake value), de los cuales los más utilizados son el SUVmax y el SUVratio ${ }^{33,34}$. El SUVmax es una medida de intensidad relativa de captación, derivada de la actividad del vóxel más captante de la lesión, corregido por la dosis inyectada, el peso del paciente, y el tiempo entre la inyección del radio-trazador y la toma de imagen ${ }^{35}$. El SUVratio es una relación entre la captación de la lesión y una captación considerada “estándar" como el pool sanguíneo o el hígado ${ }^{33}$.

Estudiando EI de válvulas protésicas, Swart y cols. ${ }^{36}$, obtiene una sensibilidad y especificidad de 75 y $86 \%$ para SUVratio $\geq 2$, y de $60 \%$ y $91 \%$ para $\operatorname{SUVmax}>4,2$. Sin embargo, no demuestran superioridad del método semi-cuantitativo respecto a la evaluación visual. Pizzi y cols., obtienen resultados similares en una serie de 92 
neran alta concentración de células inflamatorias entre la prótesis y su zona de inserción endocárdica ${ }^{1,34}$. Otro factor a considerar es que las vegetaciones son avasculares, lo que se traduce en baja captación de glucosa, y generalmente pequeñas, lo que resta sensibilidad al método. Por otro lado, el constante movimiento de las vegetaciones disminuye aún más su detectabilidad mediante PET/CT. La sensibilidad aumenta en caso de destrucción de la válvula nativa, que se suele asociar a un intenso proceso inflamatorio, más fácil de detectar por el PET/CT ${ }^{34}$.

Utilizando PET/CT en una serie de 115 pacientes con endocarditis de válvula nativa, de Camargo y cols., obtuvieron una sensibilidad (S) de $22 \%$, especificidad (E) de $100 \%$, valor predictor positivo (VPP) de $100 \%$, y valor predictor negativo (VPN) de $66 \% 34$. En una serie de 88 pacientes con sospecha de EI de válvula nativa, Kouijzer y cols. ${ }^{40}$, obtuvieron S de $30 \%$, E de $90 \%$, VPP de $91 \%$ y VPN de $27 \%$. Dado la baja S mostrada por el PET/CT, ambos autores concuerdan en que un PET/ CT normal no excluye el diagnóstico de endocarditis de válvula nativa ${ }^{12,34}$.

En pacientes con sospecha de EI de válvula nativa, el PET/CT permitiría reclasificar desde "posible" a "definitivo" a $19 \%$ de los pacientes ${ }^{34}$.

\section{PET/CT en endocarditis de válvula protésica}

El rendimiento del PET/CT es significativamente mayor en endocarditis de válvula protésica que en EI de válvula nativa, y en este contexto, es una excelente alternativa, especialmente en caso de estudios ecográficos negativos o dudosos (Figura 4). En una serie de 188 pacientes con prótesis valvular y/o de aorta ascendente, De Camargo y cols., obtuvieron una $\mathrm{S}$ de $93 \%$, E de $90 \%$, VPP de $89 \%$ y VPN de $94 \%{ }^{34}$.

En una población de 160 pacientes con válvula protésica, Swart y cols., describen una S de 74\%, E de 91\%, VPP de $89 \%$, y VPN de $78 \%{ }^{36}$. Eliminando aquellos casos con factores de confusión (adhesivo protésico, uso de antimicrobianos, proteína $\mathrm{C}$ reactiva $<40 \mathrm{mg} / \mathrm{L}$ ), el mismo autor logra mejorar significativamente el rendimiento diagnóstico del PET/CT: 91\%, 95\%, 95\%, 91\%, respectivamente.

La inclusión del PET/CT como un criterio diagnóstico de endocarditis, permite reclasificar a $76 \%$ de los pacientes con EI de válvula protésica, de "posible" a "definitivo" 34

\section{RM cerebral}

Este examen es indispensable en la evaluación de pacientes con EI, los que pueden presentar complicaciones neurológicas en 15 a 30\% de los casos, mayormente debidas a fenómenos embólicos por vegetaciones? ${ }^{7}$. En la EI o cualquier otro cuadro que pueda ocasionar embolias al sistema nervioso central, la RM cerebral 
es perentoria (Figura 5). Hay que tener presente que la embolia encefálica no es detectable con PET/CT dado la alta concentración fisiológica de ${ }^{18} \mathrm{~F}-\mathrm{FDG}$ cerebral, que suele enmascarar las lesiones ${ }^{33}$.

\section{EI asociada a dispositivos intracardiacos implantables}

El diagnóstico puede ser particularmente difícil en EI relacionados a dispositivos intracardiacos implantables (marcapasos o desfibriladores), dado que su presentación clínica es más larvada y equívoca, y el rendimiento comparativo de las técnicas imagenológicas es menor ${ }^{7}$. Al igual que en las otras EI, los hemocultivos y la ecocardiografía son los pilares del diagnóstico ${ }^{7}$. El $\mathrm{PET} / \mathrm{CT}$ con ${ }^{18} \mathrm{~F}-\mathrm{FDG}$ y el SPECT/CT con leucocitos marcados han probado tener un rol en su estudio, aunque sin evidencia suficiente como para ser incorporados dentro de los criterios diagnósticos ${ }^{7}$. El PET/ $\mathrm{CT}$ ha mostrado un alto rendimiento en la detección de infección del bolsillo del generador, con una $\mathrm{S}$ de 87 a $93 \%$, E de 93 a 100\%, VPP de 97\%, y VPN de $81 \%{ }^{30,31}$. $\mathrm{Su}$ rendimiento diagnóstico en infección del trayecto extra-cardiaco e intra-cardiaco de los electrodos es algo menor (24 a 100\%, 79 a 100\%, 66 a 100\% y 73 a 100\%, respectivamente $)^{31}$. Como ya se ha mencionado, también es útil en la detección de émbolos sépticos pulmonares, frecuentes en estos pacientes ${ }^{30}$.

\section{Hallazgos extra-cardiacos asociados a la endocarditis}

Exámenes de cuerpo completo como el PET/CT pueden mostrar positividad en patología extra-cardiaca asociada a la endocarditis. Un hallazgo frecuente es la concomitancia con embolia séptica, que se produce en 15 a $58 \%$ de los $\operatorname{casos}^{33,38}$, siendo las localizaciones más frecuentes el bazo (Figura 6), la columna vertebral y los pulmones (Figura 3).

EL PET/CT es de gran ayuda en la detección de focos infecciosos metastásicos, y debiera ser realizado en todos los casos con sospecha de $\mathrm{EI}^{40}$. Utilizando PET/CT, Kouijzer y cols. ${ }^{40}$, detectaron infección metastásica en $54 \%$ de los pacientes con EI de VN, en las siguientes ubicaciones (en orden decreciente): infección endovascular, espondilodiscitis, focos pulmonares, artritis séptica, infección de tejidos blandos, absceso esplénico y osteomielitis no vertebral.

La embolia séptica ósea merece una mención especial dado su alta frecuencia y a que los métodos radiológicos tradicionales como la radiografía y tomografía computada poseen bajo rendimiento en su detección precoz, ya que requieren una significativa destrucción de la matriz ósea para ser visibles. Para su adecuado estudio, es necesario utilizar métodos con una vocación más funcional como la RM e idealmente de cuerpo completo como el PET/

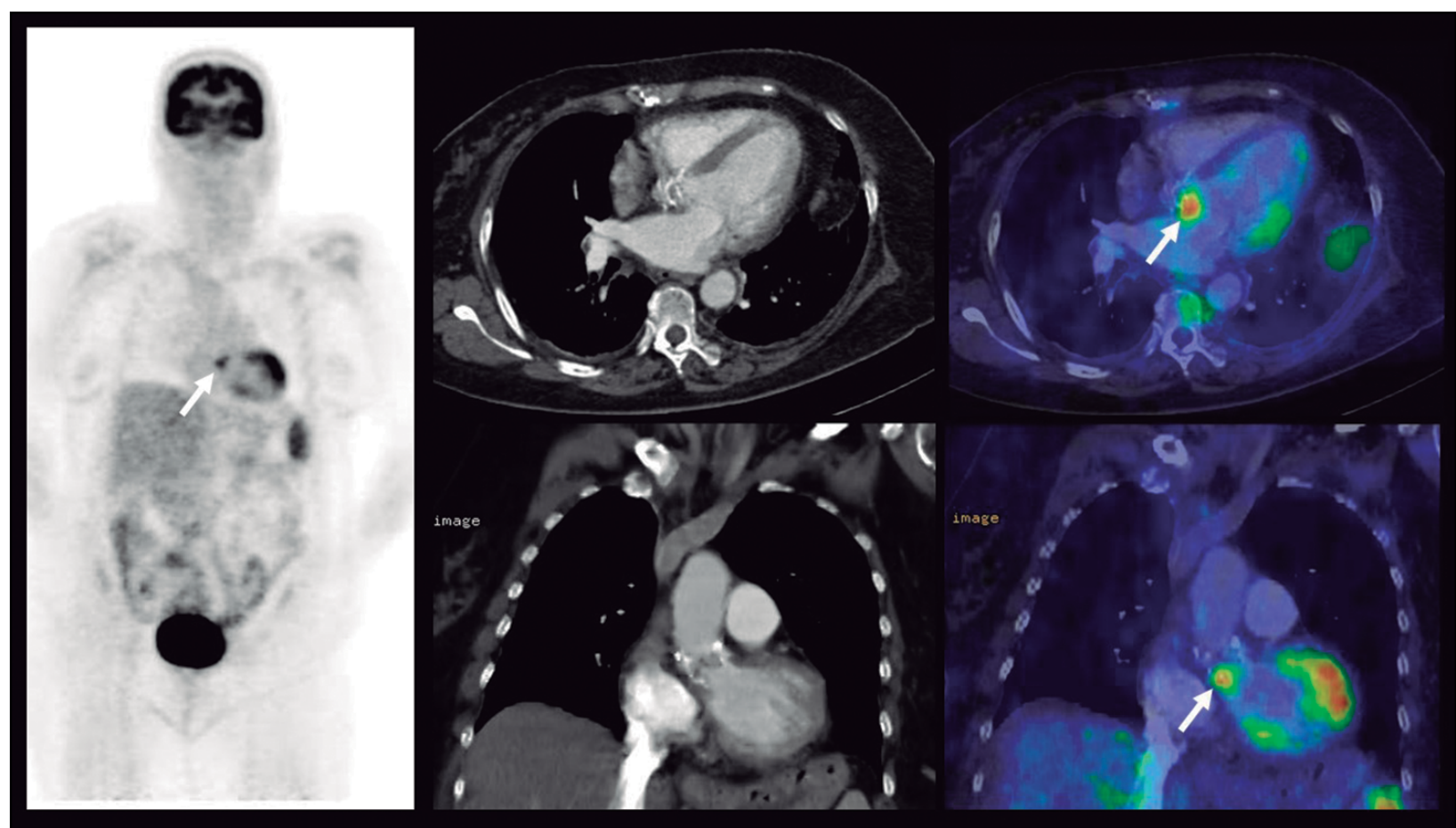

Figura 4. Mujer de 71 años portadora de prótesis valvular biológica aórtica, que comienza con fiebre y malestar general. PET/CT muestra foco intensamente hipercaptante en la zona de inserción de una prótesis valvular aórtica (flecha) con un SUVmax de 8,3 consistente con El. La ecocardiografía trans-esofágica fue normal. 


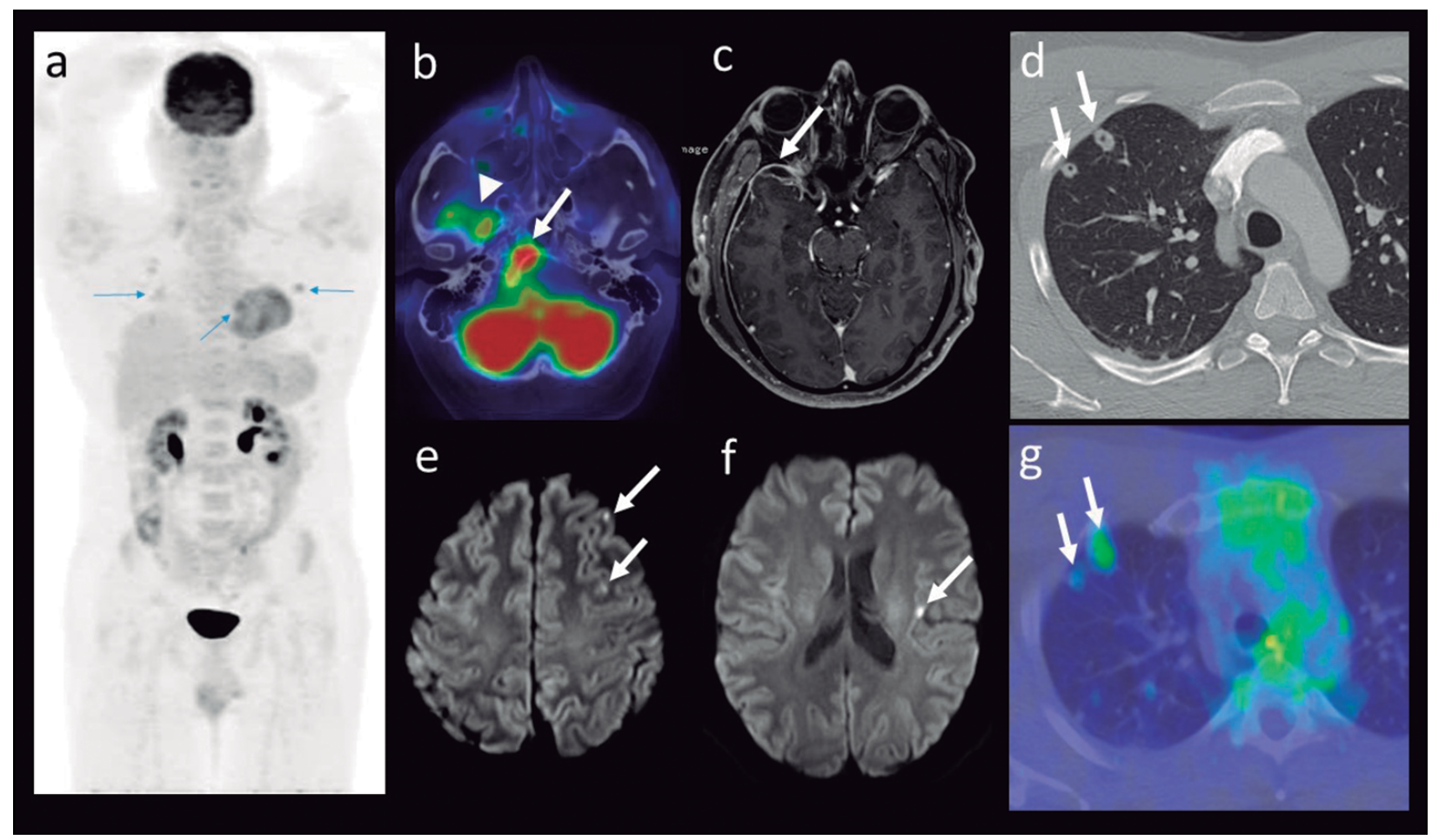

Figura 5. Hombre 37 años con historia de infección dental, inicia cuadro de tres semanas con astenia y compromiso del estado general, luego se agrega fiebre. Hemocultivos positivos para Streptococcus constellatus multisensible. Ecocardiografía TT y TE normales. a) PET/CT ${ }^{18}$ F-FDG muestra focos embólicos pulmonares e hipercaptación irregular en el plano valvular mitral (flechas). b) PET/CT demuestra compromiso óseo en la base del cráneo (flecha) y de la musculatura masticatoria a derecha (cabeza de flecha). Resonancia magnética revela empiema subdural (c) y pequeñas lesiones isquémicas frontales (e) e insular izquierda (f) posiblemente embólicas. PET/CT con pequeñas embolias pulmonares, algunas de ellas cavitadas (flechas en $\mathbf{d}$ y $\mathbf{g}$ ).

$\mathrm{CT}^{18,30}$. Las embolias articulares o de otros tejidos blandos como los músculos también son mejor visualizados con estos métodos.

Al ser considerado un criterio diagnóstico menor de endocarditis, la detección de lesiones embólicas aportaría indirectamente a su diagnóstico, permitiendo reclasificar pacientes de "posible" a "definitivo" 34 .

\section{Diagnósticos alternativos a endocarditis}

En casos en que el diagnóstico de endocarditis no se llegue a confirmar, es útil evaluar otras etiologías que expliquen el cuadro clínico. En tales casos el PET/CT puede revelar un diagnóstico alternativo en $45 \%$ de los pacientes ${ }^{34}$.

En este grupo se deben considerar 1) procesos infecciosos: neumonía, mediastinitis/osteomielitis post esternotomía (Figura 7), espondilodiscitis, aortitis infecciosa; 2) procesos inflamatorios no infecciosos: síndrome postpericardiotomía, miocarditis (Figura 8), arteritis (células gigantes y Takayasu), enfermedades del sistema retículo endotelial, sarcoidosis; y 3 ) diversas neoplasias, particularmente linfoma y cáncer de colon $^{33,34}$.

\section{Conclusión}

La EI es una patología de alta mortalidad. Su diagnóstico precoz y manejo multidisciplinario es indispensable en la tarea de mejorar la sobrevida y disminuir la morbilidad y secuelas a largo plazo. La ETT y ETE son las modalidades de imagen de elección en su diagnóstico. La TCMC cardiaca y el PET/CT ${ }^{18} \mathrm{~F}$-FDG son herramientas de gran utilidad en el diagnóstico y evaluación de complicaciones de estos pacientes, y han sido incorporados recientemente como un criterio diagnóstico mayor en las guías cardiológicas de manejo de EI. Ambos son particularmente útiles en aquellos pacientes con EI en válvula protésica, casos en que la ecocardiografía pierde rendimiento. El SPECT/CT debiera ser utilizado cuando el resto de las técnicas no son conclusivas, o no están disponibles. Dado la alta frecuencia de complicaciones embólicas en pacientes con EI, su estudio dirigido es perentorio, utilizando para este efecto RM cerebral y exámenes de cuerpo completo como el PET/CT. 


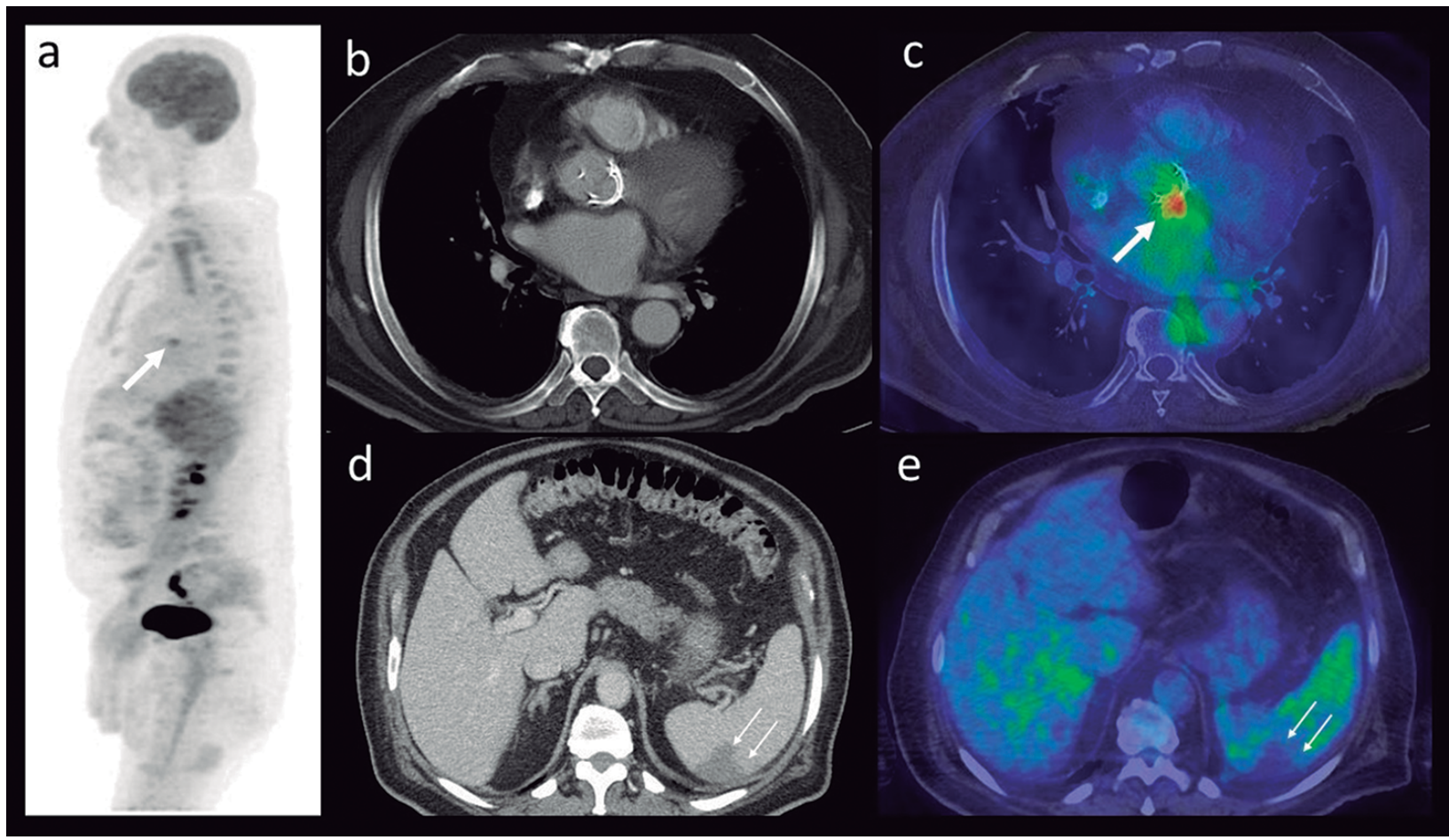

Figura 6. Hombre de 70 años portador de prótesis valvular aórtica, que comienza con fiebre y compromiso del estado general; a) MIP del PET/CT en visión lateral izquierda con foco hipercaptante proyectado a la región cardiaca (flecha); b) Corte axial de TC sin lesiones; c) PET/CT en el mismo corte axial muestra intensa hipercaptación focal paravalvular aortica con SUVmax: 6,2 (flecha) que fue confirmada como absceso perivalvular en ecocardiografía trans-esofágica; d, e) Corte axial del abdomen superior con infarto esplénico, sin concentración de ${ }^{18 F-F D G ~ e n ~ e l ~ P E T / C T ~(f l e c h a s ~ d e l g a d a s) . ~}$

Figura 7. Osteomielitis esternal. Hombre de 85 años con fiebre y compromiso del estado general. Antecedente de cirugía cardiaca e instalación de stent aortoiliaco 2 años antes. PET/CT muestra hipercaptación esternal en relación a esternotomía antigua (flechas en a y c) compatible con osteomielitis esternal; b) En TC se observa ausencia de consolidación de esternotomía con esclerosis e irregularidad de los bordes quirúrgicos; $\mathbf{d}$ y e) No hay focos hipercaptantes anormales en válvulas cardiacas, en relación al stent aorto-iliaco (cabezas de flecha blancas) ni aneurisma de arteria iliaca (cabeza de flecha amarilla).

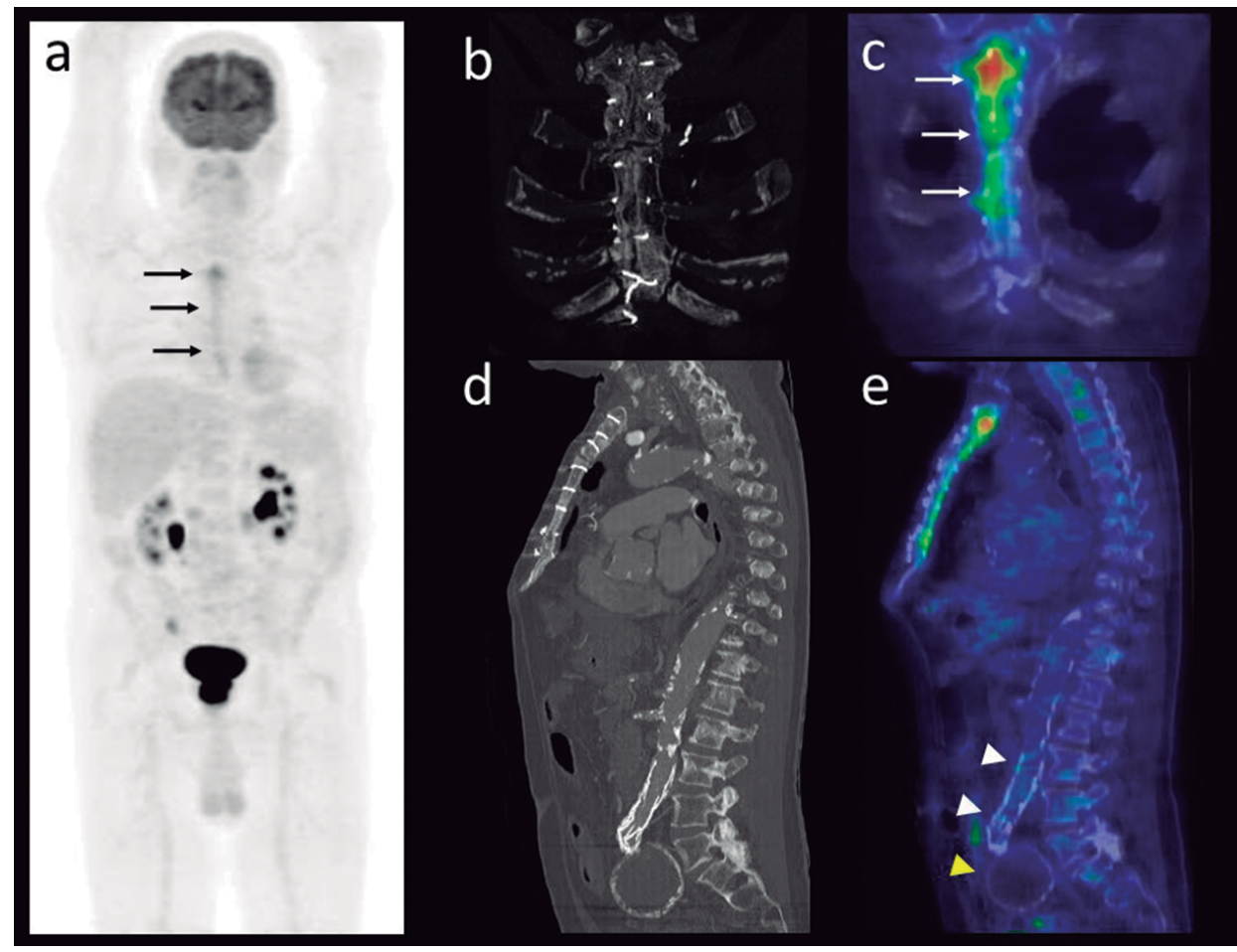




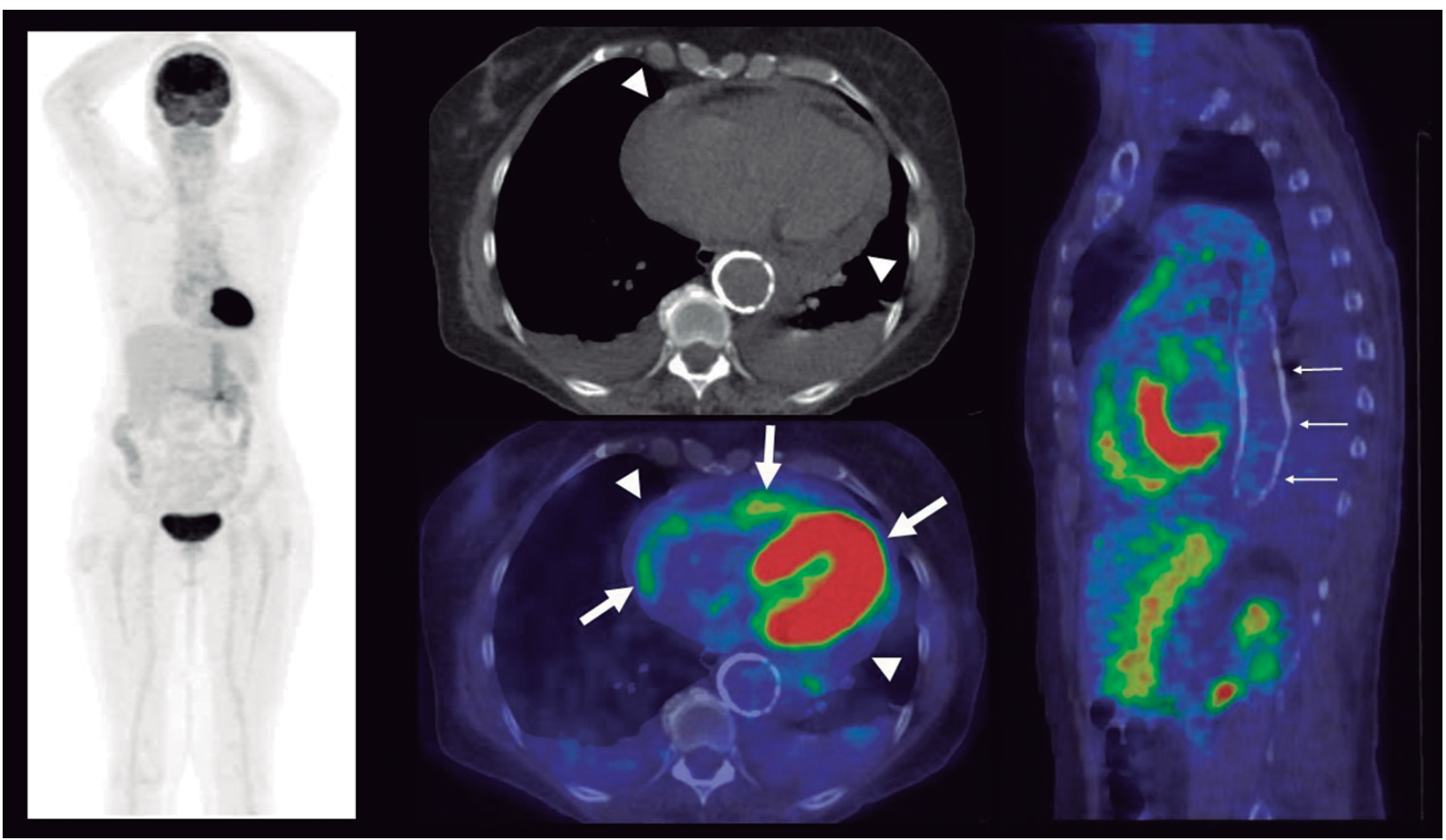

Figura 8. Miopericarditis aguda. Mujer 74 años portadora de endoprótesis aórtica con compromiso del estado general marcado, anorexia, mialgias. Ecocardiografía muestra moderada cardiomegalia, sin signos de EI. PET/CT revela hipercaptación anómala difusa del miocardio (flechas), mayor en el ventrículo izquierdo, y moderado derrame pericárdico (cabeza de flechas) de leve captación, compatible con miopericarditis. Endoprótesis aórtica sin signos de complicación (flechas delgadas).

\section{Referencias bibliográficas}

1.- Cahill T J, Prendergast B D. Infective endocarditis. Lancet 2016; 387: 882-93. doi: 10.1016/S0140-6736(15)00067-7.

2.- Duval X, Delahaye F, Alla F, Tattevin P, Obadia J-F, Le Moing V, et al. Temporal trends in infective endocarditis in the context of prophylaxis guideline modifications: three successive population-based surveys. J Am Coll Cardiol. 2012; 59: 1968-76. doi: 10.1016/j. jacc.2012.02.029.

3.- Marijon E, Ou P, Celermajer D S, Ferreira B, Mocumbi A O, Jani D, et al. Prevalence of rheumatic heart disease detected by echocardiographic screening. N Engl J Med. 2007; 357: 470-6. doi: 10.1056/ NEJMoa065085.

4.- Seckeler M D, Hoke T R. The worldwide epidemiology of acute rheumatic fever and rheumatic heart disease. Clin Epidemiol. 2011; 3: 67-84. doi: 10.2147/CLEP.S12977.

5.- Greenspon A J, Patel J D, Lau E, Ochoa J A, Frisch D R, Ho R T, et al. 16-year trends in the infection burden for pacemakers and implantable cardioverter-defibrillators in the United States 1993 to 2008. J Am Coll
Cardiol. 2011; 58: 1001-6. doi: 10.1016/j. jacc.2011.04.033

6.- Oyonarte M, Montagna R, Braun S, Rojo P, Jara J L, Cereceda M, et al. Endocarditis infecciosa: características clínicas, complicaciones y mortalidad en 506 pacientes y factores pronósticos de sobrevida a 10 años (1998-2008). Estudio cooperativo nacional en endocarditis infecciosa en Chile (ECNEI-2). Rev Med Chile 2012; 140: 1517-28. doi: 10.4067/S0034-98872012001200001.

7.- Habib G, Lancellotti P, Antunes M J, Bongiorni M G, Casalta J-P, Del Zotti F, et al. 2015 ESC Guidelines for the management of infective endocarditis. The Task Force for the Management of Infective Endocarditis of the European Society of Cardiology (ESC). Eur Heart J. 2015; 36: 3075-128. doi: 10.1093/ eurheartj/ehv319.

8.- Vilacosta I, Olmos C, de Agustín A, López J, Islas F, Sarriá C, et al. The diagnostic ability of echocardiography for infective endocarditis and its associated complications. Expert Rev Cardiovasc Ther. 2015; 13: 1225-36. doi: 10.1586/14779072.2015.1096780.

9.- Agnihotri A K, McGiffin D C, Galbraith A J, O'Brien M F. The prevalence of infective endocarditis after aortic valve replacement. J Thorac Cardiovasc Surg. 1995; 110: 1708-20. doi: 10.1016/S0022-5223(95)70035-8.

10.- Pérez-Vázquez A, Fariñas M C, GarcíaPalomo, Bernal J M, Revuelta J M, GonzálezMacías J. Evaluation of the Duke criteria in 93 episodes of prosthetic valve endocarditis: could sensitivity be improved? Arch Intern Med. 2000; 160: 1185-91. doi: 10.1001/ archinte.160.8.1185.

11.- Li J S, Sexton D J, Mick N, Nettles R, Fowler Jr V G, Ryan T, et al. Proposed modifications to the Duke criteria for the diagnosis of infective endocarditis. Clin Infect Dis. 2000; 30: 633-8. doi: $10.1086 / 313753$.

12.- Rajani R, Klein J L. Infective endocarditis: a contemporary update. Clin Med. 2020; 20: 315. doi: 10.7861/clinmed.cme.20.1.1.

13.- Kiefer T L, Bashore T M. Infective endocarditis: a comprehensive overview. Rev Cardiovasc Med. 2012; 13 (2-3): e105-e120. PMID: 23160159.

14.- Boils C L, Nasr S H, Walker P D, Couser W G, Larsen C P. Update on endocarditis-associated glomerulonephritis. Kidney Int. 2015; 87: 1241-9. doi: 10.1038/ki.2014.424

15.- Lester S J, Wilansky S. Endocarditis and 
associated complications. Crit Care Med. 2007; 35 [Suppl.]: S384-S391. doi: 10.1097/01. CCM.0000270275.89478.5F.

16.- Murillo O, Grau I, Gomez-Junyent J, Cabrera C, Ribera A, Tubau F, et al. Endocarditis associated with vertebral osteomyelitis and septic arthritis of the axial skeleton. Infection. 2018; 46: 245-51. doi: 10.1007/s15010-018$1121-9$

17.- Tamura K. Clinical characteristics of infective endocarditis with vertebral osteomyelitis. J Infect Chemother. 2010; 16: 260-5. doi: 10.1007/s10156-010-0046-8.

18.- Bergmans T, De Meester P, Herregods M C. Impact of nuclear imaging on diagnosis and management of infective endocarditis. Acta Cardiol. 2019 Apr 13; 1-5. doi: 10.1080/00015385.2019.1595268.

19.- Casella F, Rana B, Casazza G, Bhan A, Kapetanakis S, Omigie J, et al. The potential impact of contemporary transthoracic echocardiography on the management of patients with native valve endocarditis: a comparison with transesophageal echocardiography. Echocardiography 2009; 26 : 900-6. doi: 10.1111/j.1540-8175.2009.00906.x.

20.- Habib G, Badano L, Tribouilloy C, Vilacosta I, Zamorano J L, Galderisi M, et al. Recommendations for the practice of echocardiography in infective endocarditis. Eur J Echocardiogr 2010; 11: 202-19. doi: 10.1093/ ejechocard/jeq004

21.- Hansalia S, Biswas M, Dutta R, Hage F G, Hsiung M C, Nanda N C, et al. The value of live/real time three - dimensional transesophageal echocardiography in the assessment of valvular vegetations. Echocardiography 2009; 26: 1264-73. doi: 10.1111/j.1540-8175.2009.01042.x

22.- Banchs J, Yusuf S W. Echocardiographic evaluation of cardiac infections. Expert Rev Cardiovasc Ther. 2012; 10: 1-4. doi: 10.1586/ erc.11.177.

23.- Mahesh M, Cody D D. AAPM/RSNA physics of cardiac imaging with multiple-row detector CT. RadioGraphics 2007; 27: 1495-509. doi: 10.1148/rg.275075045.

24.- Machida H, Tanaka I, Fukui R, Shen Y, Ishikawa $\mathrm{T}$, Tate $\mathrm{E}$, et al. Current and novel imaging techniques in coronary CT. RadioGraphics 2015; 35: 991-1010. doi: $10.1148 / \mathrm{rg} .2015140181$

25.- Hryniewiecki T, Zatorska K, Abramczuk E,
Zakrzewski D, Szymański P, Kuśmierczyk $\mathrm{M}$, et al. The usefulness of cardiac CT in the diagnosis of perivalvular complications in patients with infective endocarditis. Eur Radiol. 2019; 29 (8): 4368-76. doi: 10.1007/s00330018-5965-2.

26.- Kim I-C, Chang S, Hong G-R, Lee SH, Lee $\mathrm{S}$, Ha J-W, et al. Comparison of cardiac computed tomography with transesophageal echocardiography for identifying vegetation and intracardiac complications in patients with infective endocarditis in the era of 3-dimensional images. Circ Cardiovasc Imaging. 2018; 11 (3): e006986. doi: 10.1161/ CIRCIMAGING.117.006986.

27.- Rouzet F, Chequer R, Benali K, Lepage L, Ghodbane W, Duval X, et al. Respective performance of $18 \mathrm{~F}-\mathrm{FDG}$ PET and radiolabeled leukocyte scintigraphy for the diagnosis of prosthetic valve endocarditis. J Nucl Med. 2014; 55: 1980-5. doi: 10.2967/ jnumed.114.141895

28.- Albano D, Bertagna F, Giubbini R. Molecular imaging in the diagnosis of infectious endocarditis-the role of PET and SPECT. Int J Cardiovasc Sci. 2020; 33: 87-93. https://doi. org/10.36660/ijcs.20190207.

29.- Signore A, Jamar F, Israel O, Buscombe J, Martin-Comin J, Lazzeri E. Clinical indications, image acquisition and data interpretation for white blood cells and antigranulocyte monoclonal antibody scintigraphy: an EANM procedural guideline. Eur J Nucl Med Mol Imaging. 2018; 45: 1816-31. doi: 10.1007/s00259-018-4052-x.

30.- Erba P A, Pizzi M N, Roque A, Salaun E, Lancellotti P, Tornos P, et al. Multimodality imaging in infective endocarditis: an imaging team within the endocarditis team. Circulation 2019; 140: 1753-65. doi: 10.1161/ CIRCULATIONAHA.119.040228.

31.- Kircher M, Lapa C. Novel noninvasive nuclear medicine imaging techniques for cardiac inflammation. Curr Cardiovasc Imaging Rep. 2017; 10: 6. doi 10.1007/s12410-017-9400-x.

32.- Harding D, Prendergast B. Advanced imaging improves the diagnosis of infective endocarditis. F1000Res. 2018; 7:F1000 Faculty Rev-674. Published 2018 May 29. doi:10.12688/f1000research.13791.1.

33.- Roque A, Pizzi M N, Cuéllar-Calàbria $\mathrm{H}$, Aguadé-Bruix S. ${ }^{18} \mathrm{~F}-\mathrm{FDG}$-PET/CT angiography for the diagnosis of infective endocarditis. Curr Cardiol Rep. 2017; 19: 15. doi: 10.1007/s11886-017-0824-3.

34.- de Camargo R A, Sommer Bitencourt M, Meneghetti J C, Soares J, Tonello Gonçalves L F, Buchpiguel C A, et al. The role of ${ }^{18} \mathrm{~F}$-fluorodeoxyglucose positron emission tomography/computed tomography in the diagnosis of left-sided endocarditis: native vs prosthetic valves endocarditis. Clin Infect Dis. 2020; 70: 583-94. doi: 10.1093/cid/ciz267.

35.- Lin E C, Alavi A, Kinahan P. Standarized uptake value. En: Lin EC, Alavi A. PET and PET/CT: A clinical guide. New York, USA: Thieme Medical Publishers Inc. 2005; 28-31.

36.- Swart L E, Gomes A, Scholtens A M, Sinha B, Lam M G E H, Tanis W, et al. Improving the diagnostic performance of ${ }^{18} \mathrm{~F}$-fluorodeoxyglucose positron-emission tomography/computed tomography in prosthetic heart valve endocarditis. Circulation 2018; 138: 1412-27. doi: 10.1161/ CIRCULATIONAHA.118.035032.

37.- Pizzi M N, Roque A, Fernández-Hidalgo N, Cuéllar-Calabria H, Ferreira-González I, Gonzàlez-Alujas M T, et al. Improving the diagnosis of infective endocarditis in prosthetic valves and intracardiac devices with ${ }^{18} \mathrm{~F}$-fluordeoxyglucose positron emission tomography/computed tomography angiography: initial results at an infective endocarditis referral center. Circulation 2015; 132: 1113-26. doi: 10.1161/ CIRCULATIONAHA.115.015316.

38.- Salomaki S P, Saraste A, Kemppainen J, Bax J J, Juhani Knuuti J, Nuutila P, et al. ${ }^{18}$ F-FDG positron emission tomography/computed tomography in infective endocarditis. J Nucl Cardiol. 2017; 24: 195-206. doi: 10.1007/ s12350-015-0325-y.

39.- Leccisotti L, Perna F, Lago M, Leo M, Stefanelli A, Calcagni M L, et al. Cardiovascular implantable electronic device infection: delayed vs standard FDG PET-CT imaging. J Nucl Cardiol. 2014; 21: 622-32. doi: 10.1007/s12350-014-9896-2.

40.- Kouijzer I J E, Berrevoets M A H, Erik H J G, Aarntzen E H J G, de Vriesc J, van Dijk A P J, et al. ${ }^{18} \mathrm{~F}$-fluorodeoxyglucose positron-emission tomography combined with computed tomography as a diagnostic tool in native valve endocarditis. Nucl Med Commun. 2018; 39: 747-52. doi: 10.1097| MNM.0000000000000864. 